\title{
GESSO COM ADIÇÃO DE RESÍDUOS COMO MATERIAL DE BAIXA COMBUSTIBILIDADE NA CONSTRUÇÃO CIVIL: UM ESTUDO TEÓRICO SOBRE A UTILIZAÇÃO, APLICABILIDADE E EFICIÊNCIA DO MATERIAL.
}

\author{
WILLYANNE FERREIRA ROCHA \\ Gestora Ambiental \\ IFCE - Campus Fortaleza \\ Ceará; Brasil \\ uille.2@gmail.com
}

\author{
ADEILDO CABRAL DA SILVA \\ Prof. Titular do IFCE - Campus Fortaleza \\ Departamento de Construção Civil/PGTGA \\ Ceará; Brasil \\ cabral@ifce.edu.br
}

\author{
JOSÉ PEREIRA \\ Engenheiro Civil \\ UNICAP \\ Pernambuco; Brasil \\ wertson120@gmail.com \\ ALENCAR, ANA \\ Engenheira Civil \\ FAP \\ Ceará, Brasil \\ karolinalencar@hotmail.com
}

\section{RESUMO}

O pó de gesso, material obtido a partir da calcinação da gipsita, apresenta uma vasta aplicação em edificações, principalmente, em virtude do seu potencial de isolamente térmico, acústico e segurança contra incêndio. De acordo com Gypsum (1999, apud LIMA, 2012), o gesso passou a ser utilizado em larga escala pelos Estados Unidos da América durante o período da $1^{\circ}$ guerra mundial, no ano de 1917, em virtude da sua resistência ao fogo. A baixa combustibilidade do material é referente a grande quantidade de moléculas de água na sua composição, fazendo com que o gesso seja empregado em construções como elemento de proteção contra o fogo. O presente trabalho aborda uma perspectiva a cerca da resistência ao fogo de matérias à base de gesso quando adicionados de resíduos, focado na análise da parte teórica com o aprofundamento do assunto, para que o artigo venha a ser de utilidade e servir como fonte de informação para outros pesquisadores. A base do trabalho consistiu no levantamento de informações presentes em livros e pesquisas realizadas na área mencionada, avaliando a aplicabilidade do material dentro da construção civil como forma de aprimorar o conhecimento relacionado ao assunto.

Palavras-chave: Gesso, Resistência ao fogo, Construção.

\section{ABSTRACT}

The gypsum powder, material resulted from gypsum calcination, presents a wide range application in building mainly for its thermal and acoustic insulation, as well as for being a fire-resistant material. According to Gypsum (1999, apud LIMA, 2012), this material was firstly used in a wide range by The United States of America during the World War I, in 1917, because of its fire resistance. The low combustible of this material is related to the amount of water molecules in its compositions, turning gypsum into a wide range used material for fire resistance in buildings. The presenting paper address a perspective related to the fire resistance of gypsum-based materials when inserted of waste materials, focusing on the theory analysis and enhancing this subject in a way to turn this survey into something useful as a source of information to other researchers. The base of this survey consisted in weighting up information from books and surveys on this topic to evaluate the material applicability in building, in a way to intend to bolster the knowledge related to this subject.

Keywords: Gypsum, Fire Resistance, Building.

\section{INTRODUÇÃO}

O Gesso, material pulverulento, branco, de baixo custo, e com características de aglomerante aéreo (endurece na presença de ar), é originado a partir da calcinação da rocha Gipsita. Historicamente apresentou uma ampla utilização em obras de arte e construção por proporcionar uma melhor qualidade em determinados aspectos, como a estética, isolamento térmico, acústico e segurança contra incêndio (ROCHA, 2018).

Atualmente o material apresenta destaque na construção civil, sendo utilizado na forma de placas ou blocos para fins de vedação (forros, paredes etc.), e para fins de acabamento, sendo aplicado como revestimento. A utilização do gesso em forma de placas e blocos são as principais formas de proporcionar o isolamento de ambientes, bem como garantir a segurança contra incêndio, visto que o gesso apresenta considerável quantidade de água em sua composição. 
As placas e blocos de gesso são fabricadas com gesso, água e aditivos, recebendo uma camada de papel cartão em cada um dos lados quando na fabricação de placas acartonadas. Essas últimas são usadas em sistema construtivo denominados de Drywall, que começou a ser usada no Brasil a partir da segunda metade da década de 1990. Apesar de seu uso por aqui ter por volta de 2 décadas, ele ainda é tímido, se compararmos com países como os EUA, que tem por volta de $90 \%$ das paredes internas residenciais constituídas desse material.

O gesso apresenta notório reconhecimento por ser um material de baixa condutividade térmica, resultando assim em um material que retarda a propagação do fogo em caso de incêndios. Devido a essa característica, o presente material fora utilizado em larga escala em diversos países durante o período da guerra. Essa característica, para além da quantidade de água no material, está relacionada a estrutura das moléculas de águas presentes no composto quando na forma sólida.

Segundo Sjöström (1992 apud TICIANI, 2005), o macrocomplexo da construção civil é um dos maiores consumidores de matérias-primas naturais, desta forma, ao longo dos anos, alternativas da produção de matérias para a construção civil por meio da reutilização foram sendo desenvolvidas. Em virtude dessa problemática da geração de resíduos causadores de intensos impactos ambientais, muitos materiais como o gesso passaram a servir como base para a reutilização desses resíduos. Contudo, as características desses resíduos adicionados podem acabar por afetar propriedades importantes do material base, como a resistência ao fogo do gesso.

O presente trabalho aborta uma perspectiva a cerca dos problemas e das possibilidades de melhoria de materias à base de gesso quando adicionados de resíudo inflamáveis, os quais estão geralmente relacionados a melhoria do isolamento acústico, térmico e a questões de sustentabilidade na construção. No decorrer desta pesquisa foi realizado um estudo teórico para o maior conhecimento sobre a problemática apresentada, de forma a analisar a abordagem do assunto no meio acadêmico.

\section{METODOLOGIA}

A metodologia do presente estudo consistiu na revisão e levantamento bibliográfico de pesquisas relacionadas à segurança contra incêndio de materiais à base de gesso com e sem adição de resíduos. Para esse fator, tópicos relacionados à segurança contra incêndio, gesso como material de segurança contra incêndio e novos materiais a base de gesso adicionados de resíduos e a segurança contra incêndio, foram consideradas como fundamentais para o embasamento e melhor entendimento a cerca do que fora considerado importante dentre os trabalhos selecionados e quantificados com relação ao tema.

\subsection{A segurança contra incêndio}

O fogo é definido como um processo químico de transformação, oxi-redução onde ocorre a combustão de materiais que apresentam características combustíveis e inflamáveis mediante a presença de um comburente, sendo esse ativado por uma fonte externa de calor, resultando na liberação de luz e calor (SOUZA, 2010).

Segundo Pérez e Cheng (2019), dentre os projetos executivos para a construção de uma edificação, encontra-se o projeto de Sistema de Segurança e Proteção Contra o Incêndio (SPCI), apresentando suma importância, visto que a ocorrência de um incêndio em uma edificação expõe os seus usuários a grande risco de vida e, em geral, causa grandes perdas patrimoniais (COSTA, 2011).

Ainda de acordo com o autor, os incêndios são fenômenos que dependem de uma série de parâmetros, apresentando relação com os seguintes fatores: a distribuição dos materiais combustíveis no interior do ambiente (carga de incêndio); grau de ventilação do ambiente (aberturas para ventilação natural); forma do compartimento (como o ambiente é dividido) e características térmicas dos materiais do compartimento (o isolamento do material utilizado).

De acordo com Zhang e Shi (2019), segurança ao fogo é um estado no qual os recursos financeiros e materiais das pessoas não são infringidas. A fim de proporcionar essa segurança, a reflexão sobre como o fogo acontece e se desenvolve é necessária para prevenir ou controlar os incêndios por meio de métodos a serem implementadas (MACNAY, et al., 2019).

As estratégias de segurança englobam todos os métodos de mitigação, soluções tecnológicas e procedimentos focados na garantia de segurança no contexto de um acidente com fogo (KARSTEN, et al., 2019). A segurança do usuário em caso de incêndio está essencialmente relacionada a forma em que a edificação é utilizada e o tempo de escape, sendo esses fatores considerados no projeto de arquitetura, principalmente, por meio do isolamento dos ambientes, evitando assim o escape da fumaça e fogo para outros compartimentos e edificações vizinhas (MEIRELLES, 2012). 
Os materiais da edificação também têm um papel importante quanto às possibilidades de desenvolvimento de um incêndio e a velocidade de propagação do fogo, pois, segundo Shoter (1962, apud MORAES, 2006), materiais inflamáveis devem ser menos utilizados por projetistas em ambientes com alto índice de risco de incêndio, a fim de proporcionar maior tempo possível aos ocupantes para a evacuação da edificação.

As propriedades e o desempenho dos materiais de revestimento contra fogo, bem como demais propriedades necessárias para garantir o desempenho e durabilidade dos materiais, devem ser determinadas por meio de ensaios realizados em laboratório nacional ou estrangeiro em concordância com a norma técnica nacional ou, na ausência desta, de acordo com norma estrangeira reconhecida internacionalmente (SEITO et al., 2008).

Tabela 1 - Lista de normas e ensaios recomendados para controle de materiais de acordo com Nunes (2008).

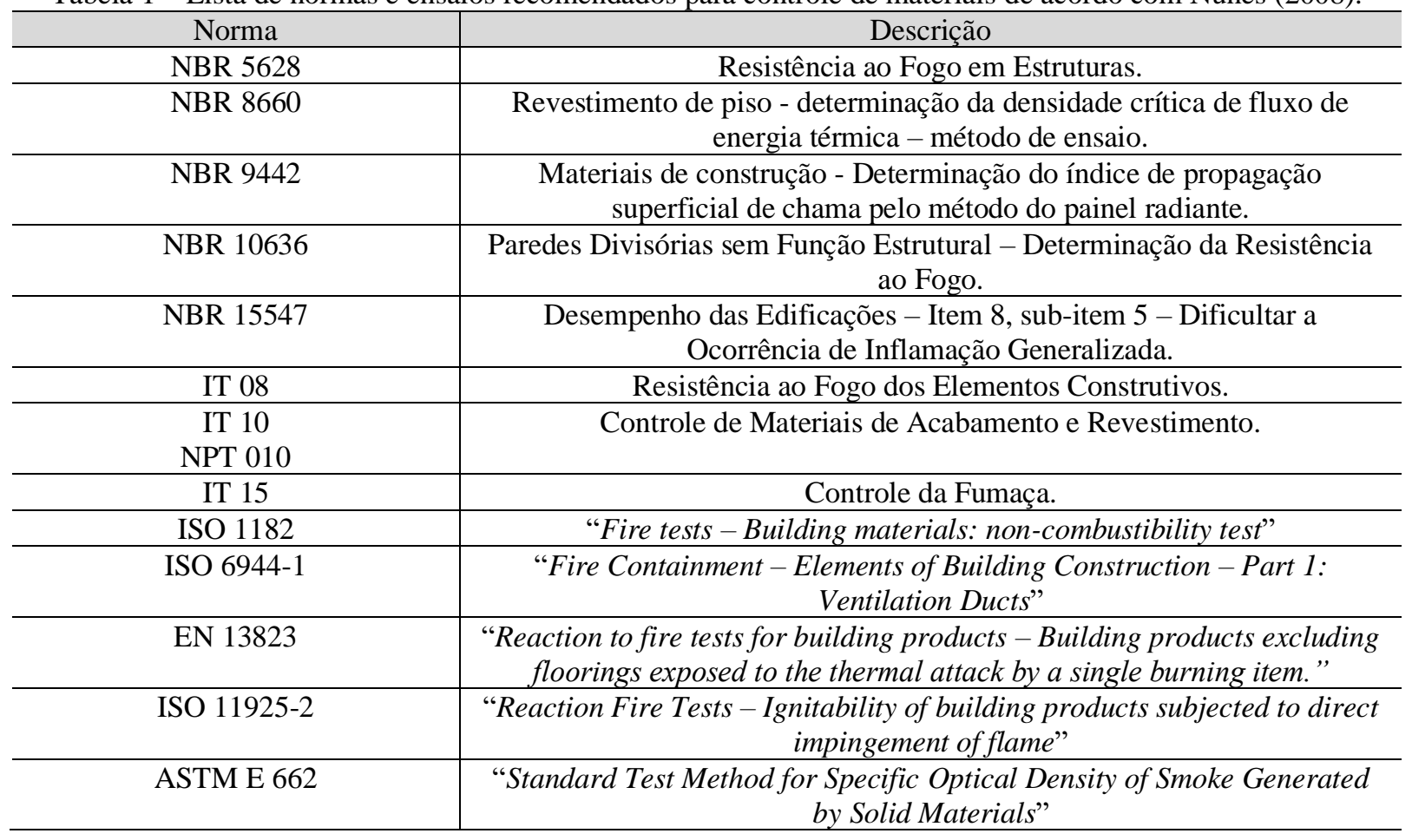

\subsection{O gesso como material de segurança contra incêndio}

$\mathrm{O}$ gesso, sulfato dihidratado de cálcio $\left(\mathrm{CaSO}_{4} \cdot 2 \mathrm{H}_{2} \mathrm{O}\right)$, tem uma vasta diversidade de utilização industrial, pois a gipsita, mineral do qual o gesso é oriundo, apresenta uma característica peculiar de fácil processo de desidratação e reidratação (BALTAR; BASTOS; LUZ 2005).

O gesso apresenta grande notoriedade devido à proteção contra a propagação de incêndio, fator que torna esse material preferível em ambientes com alto índice de risco de incêndios, como cozinhas, salas de estar e quartos, garantindo assim o tempo de escape necessário para os residentes.

Essa propriedade se deve ao fato de que o material apresenta uma concentração de água considerável em sua composição, o que segundo Lima (2012), estaria em torno de $21 \%$. Quando sujeito a calor suficiente, a água é liberada entre os poros do material e começa a evaporar. Durante esse processo a pressão dentro dos poros é aumentada e o vapor é transferido através dos poros. O calor que é absorvido durante esse processo desidrata o material, ressaltando a característica do gesso com relação a resistente ao fogo (KONTOGEORGOS; FOUNTI, 2010).

A água confinada alinhada à superfície de compósitos sólidos, no caso de placas e blocos de gesso, apresenta as ligações de hidrogênio nanométricas aumentadas em $10^{-9} \mathrm{~s}$, onde as interações com a superfície e a estrutura das moléculas fazem com que a pressão de vapor de água entre as superfícies seja inferior ao vapor de água no estado líquido (KANNO, 2009).

Em caso de incêndio, a temperatura na superfície exposta do material irá crescer uniformemente em torno de $100^{\circ} \mathrm{C}$. Nesse momento terá um retardamento na evolução da temperatura, através dos poros, enquanto a água de cristalização é 
evaporada. De acordo com a continuidade do aquecimento, os $100^{\circ} \mathrm{C}$ irá progredir aos poucos ao longo do material até que este seja completamente desidratada (KEERTHAN; MAHENDRAM, 2012).

Ainda de acordo com o autor, durante o aquecimento, o gesso passa por duas reações de desidratação, onde a primeira ocorre de $100^{\circ} \mathrm{C}$ a $120^{\circ} \mathrm{C}$, onde o sulfato de cálcio dihidratado é convertido em sulfato de cálcio hemidratado, e a segunda reação, em concordância com Andersson e Jasson (1987, apud KEERTHAN; MAHENDRAM, 2012), é a conversão de sulfato de cálcio hemidratado em anidrita a temperaturas de $210^{\circ} \mathrm{C}$ a $300^{\circ} \mathrm{C}$.

\subsection{Novos materiais a base de gesso adicionados de resíduos e a segurança contra incêndio}

A construção civil é responsável por uma grande geração de resíduos resultantes dos processos de construção e demolição, recebendo destaque pelo elevado desperdício e impacto causado ao meio ambiente (MENEZES et al., 2009). Por esse fator, materiais alternativos que sejam viáveis para uma construção mais sustentável, vêm sendo estudadas como forma de remediar muitos dos impactos resultantes da construção.

A adição de resíduos em novos materiais tem sido uma das formas mais acessíveis para mudar o viés da construção convencional, desta forma, estudos sobre a viabilidade de determinados resíduos têm sido desenvolvidos em larga escala. Contudo, análises relacionadas as possíveis problemáticas desses materiais adicionados de resíduos quando expostos a diferentes condições e ambientes não chegam a ser avaliadas de forma adequada, muitas vezes se retendo apenas a análises voltadas as propriedades físicas e mecânicas, bem como o desempenho térmico e acústico.

Parte dos resíduos utilizados na perspectiva de estudo sobre novos materiais podem apresentar certa periculosidade, estando esse inseridos dentro da presente categoria apresentada nas ABNT NBR 10006 e 10007: 2004, onde as características que definem um resíduo como perigoso são: inflamabilidade, corrosividade, reatividade, toxicidade e patogenicidade.

Estudos realizados por Huang, Liang e Hung (2010), a partir da adição de resíduo de rolhas em gesso para a decoração de ambientes, demonstram que muitas vezes o embelezamento e a melhoria no desempenho de determinados aspectos dos materiais pode acarretar uma série de problemas a segurança contra incêndio fornecida por esse. No presente estudo a resistência ao fogo do material gesso fora afetada a partir da adição do resíduo de rolha, o qual proporciona a melhoria do desempenho acústico.

Tabela 2 - Resíduos naturais e sintéticos mais adicionados a compósitos de gesso e sua inflamabilidade.

\begin{tabular}{|c|c|c|}
\hline Resíduo & Inflamabilidade & Desempenho melhorado \\
\hline Pneu & Inflamável & Isolamento acústico \\
\hline Madeira & Inflamável & Isolamento acústico \\
\hline Plástico (PET) & Inflamável & - \\
\hline EVA & Inflamável & Isolamento acústico \\
\hline Fibra Natural & Inflamável & Resistência mecânica \\
\hline Casca de ovos & Não inflamável & $\begin{array}{c}\text { Segurança contra incêndio (alta concentração de } \\
\text { Carbonato de Cálcio em sua composição) }\end{array}$ \\
\hline Vidro & Não inflamável & $\begin{array}{l}\text { Segurança contra incêndio (Fibra de vidro chega a ser } \\
\text { adicionada em indústrias como forma de aumentar a } \\
\text { resistência do gesso ao fogo) }\end{array}$ \\
\hline Vermiculita & Não inflamável & $\begin{array}{l}\text { Isolamento Térmico (Evitar o encolhimento do material } \\
\qquad \text { a altas temperaturas) }\end{array}$ \\
\hline Caulim & Não inflamável & Isolamento Térmico (Baixo condutividade térmica) \\
\hline Carbonato de Cálcio & Não inflamável & Segurança contra incêndio \\
\hline Celulose & Inflamável & Trabalhabilidade do material \\
\hline Fibras Têxteis & Inflamável & Isolamento acústico \\
\hline Resíduo de gesso & Não inflamável & $\begin{array}{c}\text { Isolamento térmico, acústico e segurança contra } \\
\text { incêndio. }\end{array}$ \\
\hline
\end{tabular}

O mesmo processo pode acontecer com demais materiais considerados inflamáveis e que proporcionam considerável melhoria no desempenho térmico, acústico e propriedades físicas e mecânicas do gesso, como poliéster, raspa de pneu e EVA, que podem acabar por ajudar na rápida propagação do calor e consequente dispersão do incêndio no ambiente. 
Segundo Hern'andez - Olivares et al., (1999 apud HUANG; LIANG; HUNG 2010), o tipo de resíduo, a granulometria, e a quantidade de gesso, são características que podem influenciar a qualidade de desempenho requerida em determinados materiais de acordo com as normas referentes, do contrário, podem afetar o desempenho referente a resistência ao fogo do material base.

\section{RESULTADOS}

A partir do levantamento de artigos relacionados a resistência contra incêndio de materiais a base de gesso convencionais, sem adição de resíduos, e não convencionais, adicionados de resíduos, em plataforma online aberta a estudantes e pesquisadores, foi selecionada uma amostra de 140 artigos de relevância obtidos a partir de pesquisas nas categorias Gypsum; Fire Resistance, para gesso convencional, e Gypsum; Waste; Fire Resistance, para gesso adicionado de resíduos ou não-convencional realizada entre os anos de 2018 e 2019.

Dessa amostra, artigos que abordaram sobre a utilização, aplicabilidade e eficiência de gesso convencional e não convencional com relação a propagação de incêndios apresentando metodologia e análises em concordância com as normas referentes ao tema, foram selecionados como convergentes a temática.

Os artigos não selecionados faziam apenas menções a propriedade de resistência ao fogo do gesso, apresentando foco no estudo sobre a utilização desse material na construção como material leve, de fácil aplicabilidade, baixo custo, possível de reciclagem e com notória qualidade como isolante de ambientes, particularmente no quesito acústico e térmico, sendo assim divergente a temática.

Após a obtenção dos dados, estes foram trabalhados e analisados em laboratório, observando-se que em materiais convencionais, de uma amostra de 140 artigos de relevância publicados entre os anos de 2018 e 2019 nas categorias referidas anteriormente, apenas 20 destes apresentaram relação direta com a temática de resistência ao fogo, de acordo com o gráfico 1 .

\section{GESSO CONVENCIONAL E RESISTÊNCIA AO FOGO}

artigos convergentes $\mathbf{n}$ Artigos divergentes

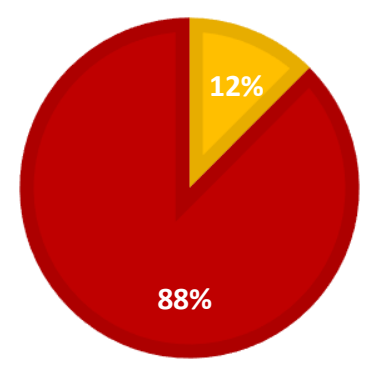

Gráfico 1 - Quantitativo do gesso convencional relacionado a resistência ao fogo.

A partir do Gráfico 1 é possível observar que dos 140 ou $100 \%$ de artigos selecionados, 120 ou $88 \%$ estão relacionados a temas divergentes a categoria pesquisada, apresentando metodologia e dados relacionados a demais análises do referido material. Apenas um percentual de 12\% dos 100\%, 20 da amostra de 140 artigos, fizeram referência direta a temática de resistência ao fogo do gesso, apresentando metodologia e dados concretos de análises realizadas em concordância com as normas referentes a resistência ao fogo.

Relacionado a materiais não convencionais, da amostra de 140 artigos de relevância publicados entre os mesmos anos anteriormente mencionados, apenas 9 destes apresentaram relação direta com a resistência ao fogo de materiais não convencionais a base de gesso, como pode ser observado no gráfico 2. 


\title{
GESSO NÃO CONVENCIONAL E RESISTÊNCIA AO FOGO
}

\author{
Artigos convergentes $\mathbf{a}$ Artigos divergentes
}

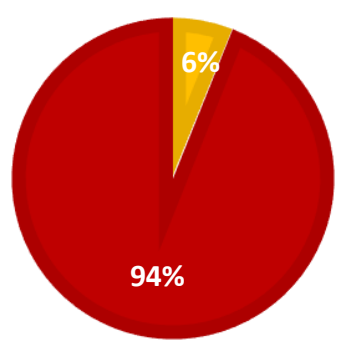

Gráfico 2 - Quantitativo do gesso não convencional relacionado a resistência ao fogo.

Dos 140 ou 100\% dos artigos selecionados, um percentual de $94 \%$ ou 131 abordaram temas divergentes a resistência ao fogo em materiais não convencionais. Um total de 9 artigos, ou 6\%, foi embasado no tema e apresentou análises em concordância com as normas para resistência ao fogo, sendo possível observar a partir do conteúdo dos trabalhos, que estas análises foram realizadas como forma de observar possível problemática gerada por resíduos inflamáveis em compósitos a base de gesso.

A partir da análise dos 9 artigos selecionados dentre os materiais não convencionais, foi possível observar que muitos destes resíduos foram adicionados, principalmente, como forma de melhorar um determinado desempenho do material, como demonstrado no texto a partir de pesquisas realizadas por Huang, Liang e Hung (2010). Sendo essa adição realizada de forma individual ou por meio da mistura de mais de um resíduo, estando entre esses: Madeira, Plástico (PET), EVA, Vidro, Fibras Têxteis, Resíduo de Gesso, Cinzas Volantes, Celulose, Escória de alto forno e FGD Gypsum.

Por meio da leitura foi possível observar também que, ao adicionar resíduos a materiais é necessário ter o conhecimento sobre o comportamento desses no presente compósito. O tipo de resíduo, a quantidade e a granulometria mostram-se como os fatores mais importantes com relação a inflamabilidade, pois durante as diferentes fases de incêndio a inflamabilidade causada pelo resíduo pode vir a acarretar a intensificação do fogo.

A quantidade de resíduo adicionado aos compósitos é um importante fator quando se considera o potencial de influência desse sobre as propriedades do material base, pois, de forma lógica, uma maior quantidade de resíduo inflamável acarreta um maior potencial de propagação de incêndio.

Dentre os demais fatores que podem afetar a propagação do fogo, a granulometria e a forma com que o resíduo é adicionado ao material são, também, fatores que podem vir a influenciar uma maior ou menor propagação do incêndio. Ao ser adicionado em forma de granulometria (fina, média ou grossa), entende-se que o resíduo pode acabar por não propagar de forma rápida o fogo, visto que esse está disperso no material que é isolante. Do contrário, preenchimentos feito entre placas de forma contínua podem acarretar uma rápida propagação do incêndio devido a continuidade do material inflamável.

Para além dos materiais utilizados na construção, também é necessário o entendimento sobre o comportamento do fogo, bem como realizar um projeto de arquitetura que considere formas de amenizar e combater a problemática dos incêndios, evitando uma propagação intensa entre os ambientes e garantindo o tempo necessário de escape para os moradores.

De forma geral, apesar de ser de suma importância estudos sobre as possibilidades da adição de resíduos em materiais, muitos destes mostram-se de forma simplificada quanto a determinação de aspectos importantes para a construção, como a segurança contra incêndio. Estudos sobre os riscos proporcionados pela adição de resíduos inflamáveis continuam por serem pouco explorados, existindo a necessidade de mais pesquisas sobre comportamento inflamável destes e como o material original pode ser afetado.

Análises relacionados as diversas problemáticas apresentadas no presente trabalho, devem ser levadas em consideração ao se estudar a possibilidade de adição de resíduos em novos materiais, para que desta forma o produto não venha a apresentar falhas relacionadas a requisitos de grande importância e que estão diretamente relacionados a segurança dos residentes. 


\section{REFERÊNCIAS}

ASSOCIAÇÃO BRASILEIRA DE NORMAS TÉCNICAS. NBR 10007: Amostragem de resíduos sólidos. Rio de Janeiro, 2004.

ASSOCIAÇÃO BRASILEIRA DE NORMAS TÉCNICAS. NBR 10006: Procedimento para obtenção de extrato solubilizado de resíduos sólidos.

BALTAR, C. A. M.; BASTOS, F. F.; LUZ, A. B. da. Gipsita. In: LUZ, A. B. da; LINS, F. A. F. Rochas \& minerais industriais: usos e especificações. 2. ed. Rio de Janeiro: CETEM/MCT, p. 449-470, 2005.

COSTA, I. A. da. Estudo Paramétrico da Resistência ao Fogo de Vigas Mistas Aço-Concreto. 2001. 95 f. Dissertação (Programa de Pós-graduação do Departamento de Engenharia Civil da Escola de Minas) - Universidade Federal de Ouro Preto, Ouro Preto, 2001.

HUANG, K. T.; LIANG, H. H.; HUNG, M. J. Improvement in fire prevention performance of cork-gypsum decorative materials by applying porous waste. International Journal of the Physical Sciences, v. 5, p. 2038-2044, 2010. Disponível em: < https://www.researchgate.net/profile/Kuo_Tsang_Huang/publication/266460208_Improvement_in_fir e_prevention_performance_of_corkgypsum_decorative_materials_by_applying_porous_waste/links/552e 78f10cf22d43 716e8a8e/Improvement-in-fire-prevention-performance-of-cork-gypsum decorative -materials-by-applying-porouswaste.pdf $>$. Acesso em: 12 dez. 2019.

KANNO, W. M. Propriedades Mecânicas do Gesso de Alto Desempenho. 2009. 130 f. Tese (Programa de Pós-graduação Interunidades em Ciência e Engenharia de Materiais) - Universidade de São Paulo, São Carlos, 2009.

KARSTEN, M. M. V.; RUGE, A. T.; HULIN, T. Closing the Gap: Merging Engineering and Anthropology in Holistic Fire Safety Assessments in The Maritime and Offshore Industries. Safety Science. v. 122, p. 104-512, fev. 2019. Disponível em:< https://www.sciencedirect.com/science/article/pii/S0925753518316 175 >. Acesso em: 15 dez. 2019.

KEERTHAN, P.; MAHENDRAN, M. Numerical studies of gypsum plasterboard panels under standard fire conditions. Fire Safety Journal, v. 53, p. 105-119, 2012. Disponível em:< https://www.researchgate.net/publica tion/257230467_Numerical_studies_of_gypsum_plasterboard_panels_under_standard_fire_conditions $>$. Acesso em: 15 dez. 2019.

KONTOGEORGOS, D.; FOUNTI, M. Numerical investigation of simultaneous heat and mass transfer mechanisms occurring in a gypsum board exposed to fire conditions. Applied Thermal Engineering. v. 30, p. 1461-1469, 2010. Disponível em:< https://www.sciencedirect.com/science/article/abs/pii/S1359431 110001134 >. Acesso em: 13 dez. 2019.

LIMA, V. C. Análise Comparativa entre Alvenaria em Bloco Cerâmico e Painéis em Gesso Acartonado para o uso como Vedação em Edifícios: Estudo de Caso em Edifício de Multipavimentos na Cidade de Feira de Santana. 2012. 66 f. Trabalho de Conclusão de Curso (Bacharelado em Engenharia Civil) - Universidade Estadual de Feira de Santana Uefs, Bahia, 2012.

MACNAY, J.; PUISA, R.; VASSALOS, D. Analysis of effectiveness of fire safety in machinery spaces. Fire Safety Journal. v. 108, p.102-859, set. 2019. Disponível em:< https://www.sciencedirect.com/science/article /abs/pii/S0379711219300864?via\%3Dihub>. Acesso em: 15 dez. 2019.

MEIRELLES, C. R. M. O Conceito de Desempenho e as Condicionais da Segurança Contra Incêndio em Projetos de Habitação de Cinco Andares no Brasil. In: $9^{\circ}$ Seminário Internacional - NUTAU 2012, $9^{\circ}$., 2012, São Paulo - SP. Anais Eletrônicos...São Paulo - SP, 2012. Disponível em: <https://www.usp.br/nutau/nutau_2012/2dia/201 20329211518_Nutau_\%20Cinco_\%20Pavimentos_revisado.pdf>. Acesso em: 20 nov. 2019.

MENEZES, R. R. et al. Reciclagem de resíduos da construção civil para a produção de argamassas. Cerâmica, São Paulo, v. 55, p. 263-270, 2009. Disponível em: <http://www.scielo.br/pdf/ce/v55n335/v55n335a05.pdf>. Acesso em: 20 nov. 2019. 
MORAES, P. D. de. Projeto de Edificações Visando à Segurança Contra Incêndio. In: ANAIS $10^{\circ}$ ENCONTRO BRASILEIRO EM MADEIRAS E EM ESTRUTURAS DE MADEIRA - EBRAMEM 2006, 10., 2006 , São Pedro-SP. Anais Eletrônicos... São Pedro-SP, 2006. Disponível em: <https://giem.ufsc.br/files/2017/02/Projeto-deedifica\%C3\%A7\%C3\%B5es-visando-a-seguran\%C3\%A7a-contra-inc\%C3\%AAndio-2006.pdf>. Acesso em: 27 nov. 2019.

NUNES, J. C. S. A Importância da Especificação dos materiais de Revestimento e Acabamento na Proteção Passiva de Incêndios. 2018. 50 f. Monografia de Especialização (Departamento Acadêmico de Construção Civil) - Universidade Tecnológica Federal do Paraná, Curitiba, 2018.

PÉREZ, C. T.; CHENG, L. Análise da Produtividade na Adequação das Instalações do Sistema de Combate a Incêndio. In: XI SIMPÓSIO BRASILEIRO DE GESTÃO E ECONOMIA DA CONSTRUÇÃO, 6º, 2019, Paraná. Anais Eletrônicos...Paraná, 2019. Disponível em:<https://www.researchgate.net/publication/336926928_ANA LISE_DA_PRODUTIVIDADE_NA_ADEQUACAO_DAS_INSTALACOES_DO_SISTEMA_DE_COMBATE_A_IN CENDIO?enrichId=rgreq-9dda7a15f6f5cb0ffee1fdda1e522a2bXXX\&enrichSource=Y292ZXJQYWdl

OzMzNjkyNjkyODtBUzo4MjAxMjk0MjAwNDYzMzZAMTU3MjU0NTQ5OTAwNw\%3D\%3D\&el=1_x_3\&_esc=pu blicationCoverPdf >. Acesso em: 28 nov. 2019.

ROCHA, W. F. Estudo do Desempenho Térmico e Acústico de Placas de Gesso Adicionadas de Resíduo de Pneu Inservível e Caulim. 2018. 63 f. Trabalho de conclusão de curso (Tecnologia em Gestão Ambiental) - Instituto Federal de Educação, Ciência e Tecnologia do Ceará, Fortaleza, 2018.

SEITO, A. I.; GILL, A. A.; PANNONI, P. N.; ONO, R.; SILVA, S. B. da.; CARLO, U. D.; SILVA, V. P e. A segurança Contra Incêndio no Brasil. 2. ed. São Paulo: Projeto Editora, 2008. 496 p.

SOUZA, M. F. de. Estudo Numérico do Isolamento Térmico de Painéis do Sistema Light Steel Framing em Situação de Incêndio. 2010. 174 f. Dissertação (Programa de Pós-Graduação em Construção Civil) - Universidade Federal de Minas Gerais, Belo Horizonte, 2010.

TICIANI, E. Racionalização de Projetos e Redução dos Custos Ambientais na Construção Civil: O Caso da Universidade das Américas - UNIAMÉRICA. 2005. 123 f. Dissertação (Programa de Mestrado em Engenharia de Produção) - Universidade Federal de Santa Catarina - UFSC, Santa Catarina, 2005.

ZHANG, L.; SHI, L. S. The Platform Design and Implementation of Campus Fire Safety Knowledge Based on Unity3D. Procedia Computer Science. v. 154, p. 832-837, jan. 2019 . Disponível em:< https://www.sciencedirect.com/science/article/pii/S1877050919308403>. Acesso em: 13 dez. 2019. 